Pacific Journal of Mathematic 


\title{
TWO THEOREMS ON GENERAL SYMMETRIC SPACES
}

\author{
H. Busemann and B. B. Phadke
}

An important result in the theory of Riemannian symmetric spaces is the theorem that the universal covering space of a complete locally symmetric space is symmetric. The proof uses the highly nontrivial property enjoyed by Riemann (but by neither Finsler nor $G$-) spaces that they are automatically analytic when locally symmetric and of class $C^{1}$. Our first theorem, nevertheless, extends the above result to locally symmetric $G$-spaces, which need not be smooth and which even when smooth are only Finsler, and not necessarily Riemann, spaces. Our second theorem states that a generic locally symmetric $G$-space is locally Minkowskian. This theorem has no analogue in Riemannian geometry.

1. Introduction. A basic theorem on symmetric Riemann spaces states that a complete locally symmetric space has a globally symmetric (or, shorter, symmetric) universal covering space. In [5] we attributed this result wrongly to Ambrose and Singer [1], who prove a more general fact; the mentioned theorem is due to Ehresmann [6] and Borel and Lichnérowicz [2].

One of the principal purposes of the present paper is extending the theorem to $G$-spoces (which are by definition complete).

Theorem I. A locally symmetric G-space has a globally symmetric universal covering space.

In view of the fact that our proof uses a simple geometric idea instead of the elaborate machinery of the literature some remarks on I are in order. A Riemann space of class $C^{1}$ is a, say, $n$-dimensional manifold with a differentiable structure and a positive definite quadratic form $d s^{2}=\sum g_{i k}(x) \xi^{i} \xi^{k}$, where $\xi=\left(\xi^{1}, \ldots, \xi^{n}\right)$ is a tangent vector and the $g_{i k}$ are of class $C^{1}$. We choose this old fashioned definition since it facilitates explaining the difference between a Riemann and a Finsler space where $d s^{2}=F^{2}(x, \xi)$ and $F$ satisfies certain standard conditions [3, § 15], in particular $F(x, k \xi)=|k| F(x, \xi)$ for real $k, F(x, \xi)>0$ for $\xi \neq 0$ and $F(x, \xi)=1$ is for fixed $x$ a strictly convex hypersurface in the tangent space instead of an ellipsoid.

A fundamental difference appears at once: in the Riemannian case $d s^{2}$ depends for fixed $x$ analytically on $\xi$. This need not be so even in the simplest (symmetric) Finsler space of class $C^{\infty}$, namely a Minkowski space whose spheres are of class $C^{\infty}$ but not analytic. 
The G-spaces comprise the Finsler spaces, but need not have any differentiability properties. It is even easy to construct Riemannian $G$-spaces, which are not class $C^{1}$, i.e., when the $g_{i k}(x)$ are merely continuous.

A basic and far from trivial step which is required by the mentioned deep methods is proving that a locally symmetric (l.s.) Riemann space of class $C^{1}$ is analytic, which implies via analytic continuation that isometries can be continued. This induced us in [5] to consider essentially only spaces without conjugate points. But we find that the simple method of [5] also works for general l.s. $G$-spaces. We construct an extension of an isometry of two possibly small domains in l.s. spaces to domains of a standard size and use this to show that continuation is possible. In several instances we could have followed Helgason's procedure [7, Chapter I, § 11, Chapter IV, §5] literally, but did not do so because the extension theorem allows certain simplifications.

In two dimensions we obtain only the Minkowski spaces in addition to known (Riemannian) symmetric spaces. However, we show with one of several available methods that in higher dimensions non-Riemannian symmetric spaces of any class and great complexity exist.

Nevertheless the result for two dimensions is in a certain sense typical, and this is the content of our second theorem. Since the difference between Riemann and Finsler spaces shows up in the line element only, a minimum of smoothness seems indispensable to formulate "generic". In a $G$-space which is continuously differentiable and regular (c.d.r) at a point $p$ in the sense of $[4, \S 5]$ a normal Minkowski metric (approximating the given metric near $p$ ) is defined. A $G$-space is generic at $p$ if it is c.d.r. at $p$ and the corresponding normal Minkowski metric admits no other true motion (i.e., different from the identity) leaving $p$ fixed than the reflection (symmetry) in $p$.

In a l.s. $G$-space if one point is generic all are; and the normal Minkowski metrics are everywhere the same. We show that the metric of the space then coincides locally or, if simply connected, globally with this common normal metric. Hence:

THEOREM II. A locally symmetric G-space which is generic at one point is locally Minkowskian.

It is therefore topologically and geodesically one of the euclidean space forms, see [3, p. 192] and, for information regarding the forms, Wolf [8]. Since a Riemann space is nowhere generic, II is a typically non-Riemannian theorem and is interesting for this 
reason alone.

2. Local extensions of isometries in locally symmetric spaces. We recall from [5] that a locally symmetric (l.s.) $G$-space is a space with a positive continuous function $\sigma(p)$ such that each $S(p, \sigma(p))$ is symmetric. From the results proved in $[5 ;(2.5)$ and (2.1) respectively] we know that each $S(p, \rho(p) / 2)$ is symmetric and that each $S(p, \rho(p) / 4)$ is symmetric and convex. It is clear that a locally isometric bijection of a convex set onto a convex set is an isometry on that convex set. This fact is useful in the following result whose proof is based on the principal idea and the method of [5, Theorem 2.5] and which is crucial for our theory. For $G$-spaces $R$ and $R^{\prime}, \kappa\left(x, x^{\prime}\right)$ stands for the number $1 / 4 \min \left(\rho(x), \rho\left(x^{\prime}\right)\right)$, $x \in R, x^{\prime} \in R^{\prime}$.

2.1. Let $R$ and $R^{\prime}$ be l.s. G-spaces and let $\phi$ be an isometry of $S(p, \alpha) \subset R$ onto $S\left(p^{\prime}, \alpha\right) \subset R^{\prime}$ with $p^{\prime}=\phi p$. Then, if $\alpha<\alpha_{0}=\kappa\left(p, p^{\prime}\right)$, $\phi$ can be extended to an isometry of $S\left(p, \alpha_{0}\right)$ onto $S\left(p^{\prime}, \alpha_{0}\right)$.

It suffices to show that if the radius $\alpha_{1}$ of the largest sphere to which $\phi$ can be extended as a local isometry is less than $\alpha_{0}$ then we reach a contradiction.

Choose $\delta$ with $\alpha_{1}<\delta<\alpha_{0}$ and $\alpha_{1}>2\left(\alpha_{1}-\delta\right)$ and on each unique $T(p, x)$ with $p x<\delta$ take $\bar{x}$ with $p \bar{x}<\alpha_{1}$. Let $\bar{x}^{\prime}=\phi \bar{x}$ and define $x^{\prime}$ by requiring that $\left(p^{\prime} \bar{x}^{\prime} x^{\prime}\right)$ and $p^{\prime} x^{\prime}=p x$. Then the association of $x$ with $x^{\prime}$ defines a continuous bijective extension of $\phi$ from $S(p, \delta)$ onto $S\left(p^{\prime}, \delta\right)$. We show that this extension is a local isometry by showing that for every $q \neq p$ with $p q<\delta$ there is a convex neighborhood $U$ of $q$ on which $\phi$ is isometric. This will provide the necessary contradiction to the definition of $\alpha_{1}$.

Define $\tau=\min \left\{\kappa\left(x, x^{\prime}\right) \mid p x \leqq \alpha_{1}, p^{\prime} x^{\prime} \leqq \alpha_{1}\right\}>0$. Choose $b$ with $(p b q), p b<\alpha_{1}, p b>b q<\tau$. Let $\psi$ be the symmetry of $S(b, \tau)$ and $\psi^{\prime}$ the symmetry of $S\left(b^{\prime}, \tau\right)$ where $b^{\prime}=\phi b$. Let $S=S(b, \gamma)$ and $S^{\prime}=S\left(b^{\prime}, \gamma\right)$ be so small that $\bar{S}$ and $\bar{S}^{\prime}$ are convex subsets of $S\left(p, \alpha_{1}\right)$ and $S\left(p^{\prime}, \alpha_{1}\right)$ respectively. Let $U$ be a convex neighborhood of $q$ so small that $U \subset S(b, \tau), \psi U \subset S\left(p, \alpha_{1}\right), \phi U=U^{\prime} \subset S\left(b^{\prime}, \tau\right), \phi^{-1} \psi^{\prime} \phi U \subset$ $S\left(p, \alpha_{1}\right)$ and such that for all $x \in U, T(p, x)$ meets $S$ in a segment $L$ and $T\left(p^{\prime}, x^{\prime}\right)$ meets $S^{\prime}$ in a segment $L^{\prime}$ with $x^{\prime}=\phi x$. Denote by $L_{0}$ and $L_{0}^{\prime}$ the prolongations of $L$ and $L^{\prime}$ respectively to $x$ and $x^{\prime}$. See Fig. 1.

On $S$ we have $\phi \psi=\psi^{\prime} \phi$ because $S$ being convex, $\phi$ is isometric on $S$. Hence $\phi L=L^{\prime}=\psi^{\prime 2} L^{\prime}=\psi^{\prime 2} \phi L=\psi^{\prime} \phi \psi L$ and this extends to $\phi L_{0}=\psi^{\prime} \phi \psi L_{0}$, so that $\phi x=\psi^{\prime} \phi \psi x$ for all $x$ in $U$. Since each of the steps $U \rightarrow \psi U \rightarrow \phi \psi U \rightarrow \psi^{\prime} \phi \psi U=U^{\prime}$ is an isometry, the restriction 
of $\phi$ to $U$ is an isometry of $U$ on $U^{\prime}$.

As observed above, this contradicts the definition of $\alpha_{1}$ and hence proves 2.1.

Note. $\kappa\left(p, p^{\prime}\right)$ is in general not nearly the best number for which 2.1 can be proved. A reader interested in this question may appreciate to know that 2.1 holds for $1 / 4 \min \left(\zeta(p), \zeta\left(p^{\prime}\right)\right)$; the definition of $\zeta(p)$ is found in $[5, \mathrm{p} .6]$.

We note also that analytic Riemann spaces $R$ and $R^{\prime}$ (even if not l.s.) always satisfy 2.1. The proof uses very strongly and essentially the analyticity assumption, see p. 62 (last six lines) and p. 63 (first four lines) in [7].

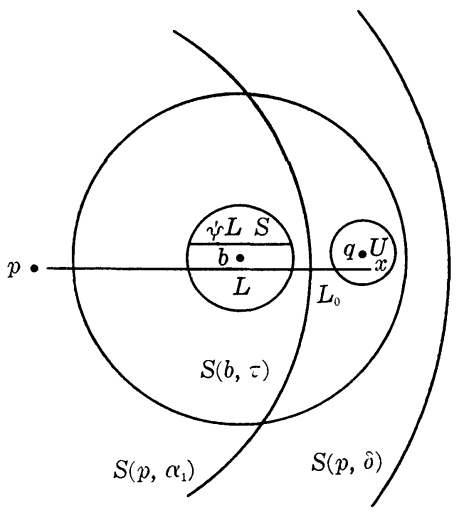

Figure 1

FIGURE 1. The figure for $S\left(p^{\prime}, \delta\right)$ is obtained by replacing the letter $y$ by $y^{\prime}$ and $\psi$ by $\psi^{\prime}$ in the above figure.

3. Continuations. In this section we prove that a simply connected l.s. $G$-space is symmetric. The proof depends on continuations of isometries along curves. Before defining this notion we note the following useful fact.

3.1. Let $T_{0}, T_{1}$ be segments from $a$ to $b$ in a simply connected, or homotopic in any, G-space. Then a deformation of $T_{0}$ into $T_{1}$ exists using curves $T_{s}$ of uniformly bounded lengths $\lambda\left(T_{s}\right)<\Lambda$.

To prove this, let $T_{0}(t), T_{1}(t)$ represent $T_{0}, T_{1}$ in terms of arc lengths $0 \leqq t \leqq a b$ and let $\gamma_{s}(t)$ be a deformation of $T_{0}$ into $T_{1}$ (so that $\left.\gamma_{0}(t)=T_{0}(t), \gamma_{1}(t)=T_{1}(t)\right)$. Partition the $t$-interval in $n$ equal parts in such a way that $\gamma_{s}\left(\left[t_{i-1}, t_{i}\right]\right)$ lies in $S\left(\gamma_{s}\left(t_{i-1}\right), \rho_{0}\right), 0 \leqq s \leqq 1$, where $\rho_{0}=\inf _{t, s} \rho\left(\gamma_{s}(t)\right)>0$ and replace $\gamma_{s} \mid\left[t_{i-1}, t_{i}\right]$ by $T_{s, t_{i}}=$ 
$T\left(\gamma_{s}\left(t_{i-1}\right), \gamma_{s}\left(t_{i}\right)\right)$. Then $\bigcup_{i=1}^{n} T_{s, t_{i}}$ is a continuous curve from $a$ to $b$ which depends continuously on $s$ and its length is less than $n \rho_{0}=\Lambda$. If $T_{s}(t)$ represents $\bigcup_{i=1}^{n} T_{s, t_{i}}$ in terms of arc length then $T_{s}(t)$ is continuous in $s$ and $t$ with $\lambda\left(T_{s}(t)\right)<\Lambda$. This proves 3.1.

Now let $R$ and $R^{\prime}$ be $G$-spaces and let $\phi$ be an isometry of a convex sphere $S \subset R$ on the (automatically convex) sphere $S^{\prime} \subset R^{\prime}$. We say that the ordered pair $(\phi, S)$ as above is an "isometry element". If $\left(\phi_{i}, S_{i}\right), i=1,2$ are two isometry elements such that $\phi_{1}=\phi_{2}$ on a nonempty open subset of $S_{1} \cap S_{2}$ then $\phi_{1}=\phi_{2}$ on $S_{1} \cap S_{2}$ since this set is convex. (Compare [3, 28.8, p. 178]). We then say that $\phi_{2}\left(\right.$ resp. $\left.\phi_{1}\right)$ is a continuation of $\phi_{1}$ (resp. $\phi_{2}$ ).

For a curve $C$ given by $\gamma(t), \alpha \leqq t \leqq \beta$ in $R$ let $\lambda>0$ and for each $t$ an isometry $\phi_{t}$ of $S(\gamma(t), 3 \lambda) \subset R$ on the sphere $S^{\prime}\left(\gamma^{\prime}(t), 3 \lambda\right) \subset R^{\prime}$ where $\gamma^{\prime}(t)=\phi_{t}(\gamma(t))$ exist such that $\phi_{t_{2}}$ is a continuation of $\phi_{t_{1}}$ if $0<t_{2}-t_{1}<\lambda$. Then $\gamma^{\prime}(t)$ is continuous. Take a partition $\Delta_{t}: \alpha=$ $t_{0}<\cdots<t_{\nu}=\beta$ of $[\alpha, \beta]$ such that for each $i$ the restriction $\gamma(t) \mid\left[t_{i}, t_{i+1}\right]$ of $\gamma(t)$ to $\left[t_{i}, t_{i+1}\right]$ lies in $S\left(\gamma\left(t_{i}\right), \lambda\right)$. Put $\phi_{t_{i}}=\phi_{i}$. If $\phi_{i}$ is a continuation of $\phi_{i-1}(i=1,2, \ldots, \nu)$ then the sequence $\phi_{0}, \cdots, \phi_{\nu}$ leads to an isometry $\phi_{C}$ of $S(b, \lambda)=S(\gamma(\beta), \lambda)$ on $S^{\prime}\left(\gamma^{\prime}(\beta), \lambda\right)=S\left(b^{\prime}, \lambda\right)$ which we call the result of continuing $\phi_{a}$ along $C$.

We must show that $\phi_{C}$ does not depend on $\Delta_{t}$. Let $\tau \in\left(t_{i}, t_{i+1}\right)$. Then $\phi_{\tau}$ continues $\phi_{i}$ and $\phi_{i+1}$ continues $\phi_{\tau}$, so the partition $t_{0}, t_{1}, \cdots$, $t_{i}, \tau, t_{i+1}, \cdots, t_{\nu}$ also leads to $\phi_{C}$. Hence generally, any partition $\Delta_{\tau}: \alpha=\tau_{0}<\cdots<\tau_{s}=\beta$ for which $\gamma(t) \mid\left[\tau_{i}, \tau_{i+1}\right] \subset S\left(\gamma\left(\tau_{i}\right), \lambda\right)$ yields $\phi_{C}$.

Let now $R$ and $R^{\prime}$ be 1.s. $G$-spaces and let $Q \subset R$ and $Q^{\prime} \subset R^{\prime}$ be compact sets. Define $\kappa=\min \kappa\left(x, x^{\prime}\right), x \in Q, x^{\prime} \in Q^{\prime}$ and $U_{x}=$ $S(x, \kappa / 3), x \in Q$. If $p \in U_{x}$ and $\phi_{p}$ is an isometry of a neighborhood of $p$ taking $p$ to $p^{\prime}, p^{\prime} \in Q^{\prime}$, then by $2.1, \phi_{p}$ can be extended to $S\left(p, \kappa\left(p, p^{\prime}\right)\right) \supset U_{x}$; because whenever $y x<\kappa / 3$, we have $y p \leqq y x+$ $x p \leqq 2 \kappa / 3<\kappa \leqq \kappa\left(p, p^{\prime}\right)$. Thus continuation of such an isometry $\phi_{p}$ is independent of the path of continuation in $U_{x}$, in the sense that, if $\phi_{0}$ and $\phi_{1}$ are two continuations of $\phi_{p}$ along paths from $p$ to $q$ lying in $U_{x}$ then $\phi_{0}=\phi_{1}$ in a neighborhood of $q$. We call such a neighborhood $U_{x}$ a "neutral" neighborhood.

Returning to homotopic segments $T_{0}$ and $T_{1}$ in $R$ with a homotopy $T_{s}(t)$ as in 3.1 let $\phi_{a}$ be an isometry of a neighborhood of $a$ with $a^{\prime}=\phi_{a} a$. We show that continuation along each $T_{s}(t)=\gamma(t)$, $0 \leqq t \leqq \alpha$, is possible. Put $\kappa=\min \kappa\left(x, x^{\prime}\right), x \in S(a, \Lambda), x^{\prime} \in S\left(a^{\prime}, \Lambda\right)$. Choose $\Delta_{t}: 0 \leqq t_{0}<t_{1} \cdots<t_{m}=\alpha$ with $\gamma\left(t_{i}\right) \gamma\left(t_{i-1}\right)<\kappa / 3$ and put $\gamma_{i}=\gamma(t)\left[t_{i-1}, t_{i}\right]$. By 2.1 and the definition of $\kappa, \phi_{a}$ can be continued along $\gamma_{1}$ getting $\gamma_{1}^{\prime}$ with the length $\lambda\left(\gamma_{1}\right)=\lambda\left(\gamma_{1}^{\prime}\right)$ and $\gamma^{\prime}\left(t_{1}\right)=$ $\phi_{t_{1}}\left(\gamma\left(t_{1}\right)\right)$. Similarly $\phi_{t_{2}}$ can be continued along $\gamma_{2}$ getting $\gamma_{2}^{\prime}$ with $\lambda\left(\gamma_{2}^{\prime}\right)=\lambda\left(\gamma_{2}\right)$ and $\gamma^{\prime}\left(t_{2}\right)=\phi_{t_{2}}\left(\gamma\left(t_{2}\right)\right)$. This process yields a continuation of $\phi_{a}$ along $\gamma(t)$ because for all $j \leqq m$ we have $a^{\prime} \gamma_{j}^{\prime}\left(t_{j}\right) \leqq \sum_{i=1}^{j} \lambda\left(\gamma_{i}^{\prime}\right)<\Lambda$. 
If necessary refine the subdivision of the deformation square, so that the pre-image of every subsquare lies in some neutral $S(x, \kappa / 3)$. A familiar argument shows that the deformation of $T_{0}$ into $T_{1}$ can be obtained by successively traversing the boundaries of the subsquares in a suitable order, hence continuation along $T_{0}$ and $T_{1}$ leads to the same result.

Specializing further, let $R=R^{\prime}$ be l.s. and simply connected. Choose $p$ arbitrarily and let $\phi_{p}$ be the symmetry in $p$ of $S=$ $S(p, \rho(p) / 4)$. For any $x$, let $T$ be a segment from $p$ to $x$ and continue $\phi_{p}$ along $T$ to $\phi_{T}$. Then as noted above $\phi_{T}$ does not depend on the choice of segment $T$ from $p$ to $x$ and hence this defines a map $\psi$ of $R$ into itself which maps $S\left(x, \kappa\left(x, x^{\prime}\right)\right)$ isometrically on $S\left(x^{\prime}, \kappa\left(x, x^{\prime}\right)\right)$ where $x^{\prime}=\phi_{T} x$. Moreover $\psi$ coincides with $\phi_{p}$ on $S(p, \rho(p) / 4)$. Thus $\psi$ is a local isometry of $R$ into itself. By construction it maps any half geodesic issuing from $p$ on the entire opposite half geodesic, so $\psi R=R$. Since $\psi \neq I$ and $\psi^{2}=I$ in $S(p, \rho(p) / 4)$ and hence in $R$, it must be the symmetry of $R$ in $p$. Therefore we have:

Theorem I. A locally symmetric G-space has a globally symmetric universal covering space.

Applying our result $[5,3.5]$ we find the corollary:

3.2. If a positive continuous function $\lambda(p)$ exists on the $G$-space $R$ such that for each $p$ all ellipses in $S(p, \lambda(p))$ (i.e., the loci $\left.f_{1} x+x f_{2}=\beta>f_{1} f_{2}>0\right)$ have centers, then the universal covering space of $R$ is globally symmetric.

4. Examples. We now show that when the (topological) dimension of the space exceeds 2 there are lots of nontrivial examples of locally symmetric spaces.

One method of generating many is the following. Let $g(u)=$ $g\left(u_{1}, u_{2}, \cdots, u_{s}\right)$ be defined for $u_{i} \geqq 0, g(u)>0$ for $u \neq 0, g(u)<$ $g(v)$ if $u_{i} \leqq v_{i}$ for all $i$ and $u_{j}<v_{j}$ for at least one $j, 1 \leqq i, j \leqq s$. Moreover let $g(u)$ be positive homogeneous, i.e., $g(k u)=k g(u)$ for $k \geqq 0$, and assume that $g(u+v)<g(u)+g(v)$ unless $u=v=0$ or $u=\lambda v$ for a scalar $\lambda$. An example of such a $g(u)$ is $g(u)=$ $\left[\sum_{i=1}^{s}\left(u_{i}\right)^{\alpha}\right]^{1 / \alpha} ; \alpha>1$.

If $R_{1}, R_{2}, \cdots, R_{s}$ are $G$-spaces then $R=R_{1} \times R_{2} \times \cdots \times R_{s}$ with points $x=\left(x_{1}, x_{2}, \cdots, x_{s}\right), x_{i} \in R_{i}$ and distance $x y=g\left(x_{1} y_{1}, x_{2} y_{2}, \cdots\right.$, $\left.x_{s} y_{s}\right)$ is a $G$-space. If $x_{i}(t)$ is a geodesic in $R_{i}$ then $x(t)=\left(x_{1}(t), \ldots\right.$, $\left.x_{s}(t)\right)$ is a geodesic in $R$; the first $t$ need not be arc length for $x(t)$ but $g(t, t, \cdots, t)$ is. (Compare [3, 8.15], p. 42.) 
If $\phi_{i}$ is a motion of $R_{i}$ then $\phi x=\left(\phi_{1} x_{1}, \phi_{2} x_{2}, \cdots, \phi_{s} x_{s}\right)$ is one of $R$. In particular, if each $R_{i}$ is either locally symmetric or symmetric then so is $R$.

5. Generic locally symmetric spaces. We begin with the following observation on l.s. $G$-spaces.

5.1. In a l.s. G-space two points, $p$, $q$ have for a suitable $\rho>0$ isometric neighborhoods $S(p, \rho)$ and $S(q, \rho)$.

This can be seen either by applying our Theorem I or from the following direct argument: Let $\sigma=\min \{\sigma(x) \mid x \in a$ segment $T=$ $T(p, q)$ joining $p$ to $q$ \}. By choosing points $p=p_{0}, p_{1}, \cdots, p_{k}=q$, $k=2 l-1$, in this order on $T$ such that $p_{i} p_{i+1}<\sigma / 4$ and denoting by $\psi_{i}, i=1,2, \cdots, l$, the symmetry of $S\left(p_{2 i}, \sigma\right)$ we see that $\psi_{r}$ maps $S\left(p_{2 r-1}, \sigma / 2\right)$ isometrically on $S\left(p_{2 r+1}, \sigma / 2\right)$. Hence $\phi=\psi_{l} \psi_{l-1} \cdots \psi_{1}$ maps $S(p, \sigma / 2)$ isometrically on $S(q, \sigma / 2)$.

The notion of a continuously differentiable and regular (c.d.r.) $G$-space is defined in the following way, see [4, $\S 5]$. Denote, for $0 \leqq \beta \leqq 1$ and a unique segment $T(\alpha, q)$, by $a_{q \beta}$ the point on $T(a, q)$ with $q a_{q \beta}=\beta q a$. The space is said to be continuously differentiable at $p$ if for $\varepsilon>0$ there exists $0<\delta_{1}(\varepsilon) \leqq \rho(p)$ such that for $a, b, q$ in $S\left(p, \delta_{1}(\varepsilon)\right),\left|a_{q \beta} b_{q \beta}-\beta b a\right| \leqq \varepsilon \cdot \beta a b$ for $0 \leqq \beta \leqq 1$. The limit $a_{p \beta} b_{p \beta}: \beta$ as $\beta \rightarrow 0_{+}$then exists (denoted $m_{p}(\alpha, b)$ ) for $a, b \in S(p, \rho(p)$ ). Then $m_{p}(a, b)$ defines in $S(p, \rho(p))$ a metric which is equivalent to $a b$ and satisfies $m_{p}(a, b): a b \rightarrow 1$ when $a \neq b, a \rightarrow p, b \rightarrow p$. The space is, in addition, regular if continuation is unique for $m_{p}$, i.e., if the three points $a, b, c_{i}$ in $S(p, \rho(p))$ are distinct $(i=1,2)$ and $m_{p}(a, b)+$ $m_{p}\left(b, c_{i}\right)=m_{p}\left(a, c_{i}\right) \quad$ with $m_{p}\left(b, c_{1}\right)=m_{p}\left(b, c_{2}\right)$ imply $c_{1}=c_{2}$. Then $m_{p}(a, b)$ is a part of a Minkowski metric (with strictly convex spheres) and is called the local normal Minkowski metric at $p$ and the space the l.n. Minkowski space.

Hence we have from 5.1.

5.2. If a l.s. space is c.d.r. at one point then it is so at all points and the normal Minkowski metrics are all isometric.

Another consequence of 5.1 is:

5.3. If a l.s. space has a sphere $S(p, \rho)$ which admits for some small $\rho>0$ no other true isometry on itself, keeping $p$ fixed, than the symmetry in $p$, then the same holds for all points.

From the definition of $m_{p}$ and 5.3 we see: 
5.4. If there are two different true isometries of $S(p, \rho)$ on itself in a l.s. space and the space is c.d.r. then the normal Minkowski metric has at $p$ (or everywhere) true motions other than the symmetry in $p$.

We are now ready to prove:

IIa. If a locally symmetric G-space contains a point $p$ such that no $S(p, \rho), \rho>0$, admits a true isometry, except the symmetry in $p$, on itself keeping $p$ fixed, then the space is locally Minkowskian.

From 5.3 we see that the hypothesis of IIa holds at all points $p$. Thus, since the universal covering space of a locally symmetric space and also the universal covering space of a locally Minkowsian space is symmetric, it suffices to consider the case when the given space is globally symmetric.

Let $a$ be an arbitrary given point of the space $R$ and let $\alpha=$ $\rho(a) / 32$. Let $q_{0}, q_{1}, r$ be any three noncollinear points in $S(a, \alpha)$.

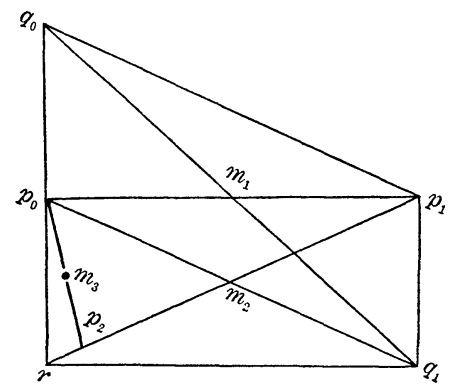

Figure 2

Let $p_{0}, m_{1}, m_{2}$ be the midpoints of the unique segments $T\left(q_{0}, r\right)$, $T\left(q_{0}, q_{1}\right)$ and $T\left(p_{0}, q_{1}\right)$ respectively. Denoting by $\psi_{i}$ the symmetry of $R$ in $m_{i}, i=1,2$, define $p_{1}=\psi_{1} p_{0}$ and $p_{2}=\psi_{2} p_{1}$. Then the segment $T\left(p_{0}, p_{2}\right)$ is also unique (since an easy computation shows that $\left.a p_{0}, a p_{2}<\rho(a)\right)$, let $m_{3}$ be the midpoint of $T\left(p_{0}, p_{2}\right)$ and let $\psi_{3}$ denote the symmetry of $R$ in $m_{3}$. See Fig. 2.

Now $\psi_{1} p_{0}=p_{1}$, so that $p_{0} m_{1}=m_{1} p_{1}=1 / 2 \cdot p_{0} p_{1}$. Since $\psi_{2} p_{1}=p_{2}$, we have $\psi_{2} \psi_{1} q_{0}=p_{0}, p_{0} p_{1}=\psi_{2} p_{0} \psi_{2} p_{1}=q_{1} p_{2}=2 p_{0} m_{1}$. As $\psi_{3} p_{2}=p_{0}$ we have $\psi_{3} \psi_{2} \psi_{1} p_{0}=p_{0}$. Hence by the hypothesis of the statement IIa, $\phi=\psi_{3} \psi_{2} \psi_{1}$ is the symmetry in $p_{0}$; or $\phi q_{0}=r$. On the other hand $\phi q_{0}=\psi_{3} \psi_{2} \psi_{1} q_{0}=\psi_{3} \psi_{2} q_{1}=\psi_{3} p_{0}=p_{2}$. Thus $p_{2}=r$. Consequently $2 p_{0} m_{1}=r q_{1}$.

This means that the triangle $q_{0} r q_{1}$ satisfies the condition of zero 
curvature [3, p. 237]. As this was an arbitrary triangle in $S(a, \alpha)$ we have proved that $R$ has zero curvature. The result IIa now follows from $[3,39.12$, p. 261$]$.

We say that a $G$-space $R$ is generic at a point $p$ if $R$ is c.d.r. at $p$ and the normal Minkowski metric at $p$ possesses no other true motions leaving $p$ fixed than the symmetry in $p$. It follows from 5.2 that:

5.5. If a l.s. space is generic at one point then this is so at all points.

Combining IIa, 5.2, 5.4, and 5.5 yields our second theorem:

THEOREM II. If a locally symmetric G-space is generic at one point then it is locally Minkowskian.

It should be noted that, actually, with a minor reinterpretation the proof for IIa works directly for II also.

Both II and IIa are definitely non-Riemannian statements. II, as mentioned in the introduction, since a Riemann space is nowhere generic and IIa because, if the space were Riemannian, it would be Euclidean, which contradicts the hypothesis of IIa.

But it is very easy to construct a Riemann (hence nowhere generic) space which is symmetric at a point $p$, but no $S(p, \rho)$ has any other true isometries leaving $p$ fixed than the symmetry in $p$.

It is also simple to produce a Finsler space, symmetric at a point $p$, where all normal metrics are isometric, but no other true motions than the symmetry in $p$ exist.

We indicate briefly how this can be done. In an $(x, y)$-plane define $F(0,0)$ as a strictly convex curve of class $C^{\infty}$ with $(0,0)=p$ as center. By an affinity $\psi(x, y)$ given by the matrix $\left(a_{i k}(x, y)\right)$ of of its coefficients transform $F(0,0)$ into $F(x, y)$. We can choose $a_{i k}(x, y)$ such that they are of class $C^{\infty}$ and $F(-x,-y)$ originates from $F(x, y)$ by $x^{\prime}=-x, y^{\prime}=-y$.

Take the $F(x, y)$ as the (infinitesimal or) local unit circles of a Finsler metric with line element $f(x, y, d x, d y)$. This will be of class $C^{\infty}$ except for $d x=d y=0$. Since the $F(x, y)$ are affinely related the local Minkowski metrics are isometric, see [3, §17]. Clearly the freedom in the choice of the $a_{i k}(x, y)$ allows us to prevent that the space has another true motion than the symmetry in $p$, or even that any $S(q, \rho)$ possesses a true isometry on itself.

The second named author thanks the University of Auckland for the use of its facilities during his visit overlapping the preparation of this paper. 
Note added in proof. In the meantime the paper by V. N. Berestovskii “On homogeneous Busemann G-spaces," Doklady Akad. Nauk, 247 (1979), No. 3 has come to our attention which states without proof a theorem basically identical with our Theorem I.

\section{REFERENCES}

1. W. Ambrose and I. M. Singer, On homogeneous Riemannian manifolds, Duke Math. J., 25 (1958), 647-699.

2. A. Borel and A. Lichnérowicz, Espaces Riemanniens et Hermitiens symétriques, C. R. Acad. Sci. (Paris), 234 (1952), 2332-2334.

3. H. Busemann, The Geometry of Geodesics, Academic Press, New York, 1955.

4. - Recent Synthetic Differential Geometry, Ergeb. Math. Band 54, SpringerVerlag, New York, 1970.

5. H. Busemann and B. B. Phadke, Symmetric Spaces and Ellipses, to appear in the Proceedings International Christoffel Symposium Birkhäuser, Basel, 1980.

6. C. Ehresmann, Sur les espaces localement homogènes, Enseignement Mathématique, 35 (1936), 317-333.

7. S. Helgason, Differential Geometry and Symmetric Spaces, Academic Press, New York, 1962.

8. J. A. Wolf, Spaces of Constant Curvature, McGraw-Hill, New York, 1967.

Received June 15, 1979 and in revised form October 29, 1979.

The University of Southern California,

Los Angeles, CA 90007

The Flinders University of South Australia,

Bedford Park, Australia

AND

The University of Auckland,

Auckland, New Zealand 


\section{PACIFIC JOURNAL OF MATHEMATICS}

\section{EDITORS}

DONALD BABBITT (Managing Editor)

University of Galifornia

Los Angeles, California 90024

Hugo RossI

University of Utah

Salt Lake City, UT 84112

C. C. MOORE AND ANDREW OGG

University of California

Berkeley, CA 94720
J. DUGUNDJI

Department of Mathematics University of Southern California Los Angeles, California 90007

R. Finn AND J. Milgram Stanford University Stanford, California 94305

\section{ASSOCIATE EDITORS}

R. ARENS

E. F. BECKENBACH

B. H. NEUManN

F. WOLF

K. YOSHIDA

\section{SUPPORTING INSTITUTIONS}

UNIVERSITY OF ARIZONA

UNIVERSITY OF BRITISH COLUMBIA CALIFORNIA INSTITUTE OF TECHNOLOGY

UNIVERSITY OF CALIFORNIA

MONTANA STATE UNIVERSITY

UNIVERSITY OF NEVADA, RENO

NEW MEXICO STATE UNIVERSITY

OREGON STATE UNIVERSITY
UNIVERSITY OF OREGON

UNIVERSITY OF SOUTHERN CALIFONIA

STANFORD UNIVERSITY

UNIVERSITY OF HAWAII

UNIVERSITY OF TOKYO

UNIVERSITY OF UTAH

WASHINGTON STATE UNIVERSITY

UNIVERSITY OF WASHINGTON 


\section{Pacific Journal of Mathematics}

\section{Vol. 92, No. $1 \quad$ January, 1981}

Michael E. Adams and J. Sichler, Lattices with unique complementation . ....1

Walter Allegretto, Positive solutions and spectral properties of second order

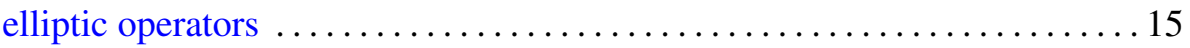

Philip J. Boland and Sean Dineen, Holomorphy on spaces of distribution . . 27

Duncan Alan Buell, Philip A. Leonard and Kenneth S. Williams, Note on

the quadratic character of a quadratic unit $\ldots \ldots \ldots \ldots \ldots \ldots \ldots \ldots \ldots$

Herbert Busemann and Bhalchandra B. Phadke, Two theorems on

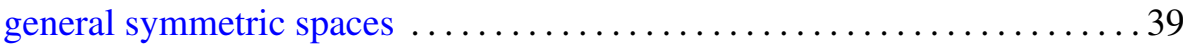

Emeric Deutsch, Bounds for the Perron root of a nonnegative irreducible

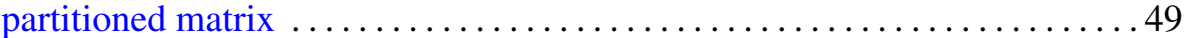

Charles F. Dunkl, A difference equation and Hahn polynomials in two

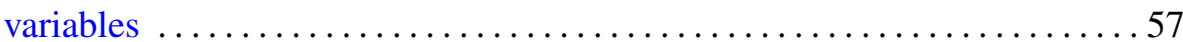

Gustave Adam Efroymson, The Riemann mapping theorem for planar Nash

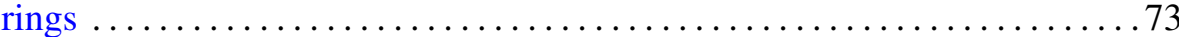

John Albert Fridy and Robert Ellis Powell, Tauberian theorems for matrices generated by analytic functions

Denton Elwood Hewgill, John Hamilton Reeder and Marvin Shinbrot,

Some exact solutions of the nonlinear problem of water waves .......887

Bessie Hershberger Kirkwood and Bernard Robert McDonald, The

symplectic group over a ring with one in its stable range

Esther Portnoy, Transitive groups of isometries on $H^{n}$

Jerry Ridenhour, On the sign of Green's functions for multipoint boundary

value problems

Nina M. Roy, An $M$-ideal characterization of $G$-spaces

Edward Barry Saff and Richard Steven Varga, On incomplete

polynomials. II

Takeyoshi Satō, The equations $\Delta u=P u(P \geqq 0)$ on Riemann surfaces and

isomorphisms between relative Hardy spaces

James Henry Schmerl, Correction to: "Peano models with many generic classes"

Charles Madison Stanton, On the closed ideals in $A(W)$

Viakalathur Shankar Sunder, Unitary equivalence to integral operators

Pavel G. Todorov, New explicit formulas for the $n$th derivative of composite functions

James Li-Ming Wang, Approximation by rational modules on boundary sets

Kenneth S. Williams, The class number of $Q(\sqrt{p})$ modulo 4 , for $p \equiv 5$

$(\bmod 8)$ a prime 